\title{
Cross-tail velocity component in the plasma sheet fast flows
}

\author{
N. P. Dmitrieva \\ Institute of Physics, University of St-Petersburg, St-Petersburg, Russia \\ Received: 22 May 2007 - Revised: 3 December 2007 - Accepted: 18 December 2007 - Published: 11 June 2008
}

\begin{abstract}
The flux transfer in the magnetotail plasma sheet is mainly provided by the tail-aligned fast plasma flows (Bursty Bulk Flows - BBFs). In this paper we study the events with a large cross-tail velocity component, including their occurrence and relationship to the standard BBFs. We found out that a significant part of large $V_{y}$ events are a subgroup connected with the BBFs propagation. The maximal deviation of the velocity vector from the $\mathrm{X}$ direction (about 40-50 degrees, on average) is observed near the BBFs' leading front in the sheath, where the fast flow interacts with surrounding plasma. The average variation of the velocity direction in the vicinity of the BBF resembles a plasma vortex. Our results support the model, in which the BBF represents a polarized, bubble-like flux tube, propagating through the plasma sheet.
\end{abstract}

Keywords. Magnetospheric Physics (magnetotail; plasma convection) - Space plasma physics (transport processes)

\section{Introduction}

Interest in the fast plasma flows in the magnetospheric plasma sheet has been growing during the last decade. This is because the BBFs have probably a close relationship to the magnetic field reconnection in the tail and also due to their key role in the magnetotail transfer (Baumjohann et al., 1990; Baumjohann, 2002; Angelopoulos et al., 1994). Indeed, as known, the BBFs provide from 50\% (Schödel et al., 2001) up to $80 \%$ (Angelopoulos et al., 1994, 1996) of the plasma and magnetic flux transport.

Previous studies revealed and described the average characteristics of the fast plasma flows (Angelopoulos et al., 1994; Ohtani et al., 2004). The main properties of the BBF proper are: (1) the plasma density and pressure are

Correspondence to: N. P. Dmitrieva

(dmitrieva@geo.phys.spbu.ru) reduced, (2) the temperature is enhanced; (3) the magnetic field displays the bipolar $B_{z}$ variation and a $\left|B_{x}\right|$ decrease. These characteristics are in agreement with the BBF being a plasma-depleted, electrically-polarized plasma tube (plasma bubble). A simple analytical model for the bubble propagation has been developed by Pontius and Wolf (1990), Chen and Wolf, (1993), whereas a pattern of the plasma flow near the bubble has been obtained among other results in the MHD simulation by Birn et al. (2004). At geocentric distances $>15 R_{E}$ near midnight (where the BBFs are most frequently observed) the flux tube plane lies near the XZ plane, and the velocity direction at the plasma sheet should nearly coincide with the $\mathrm{X}$ axis. Indeed, this was confirmed by the experimental data by Angelopoulos et al. $(1992,1994)$.

However, the results of Baumjohann et al. (1990) reveal a considerable $V_{y}$ velocity component in the fast flows at the distances $<20 R_{E}$. Particularly, the velocity vector deviation from the tail axis is more than 34 degrees in $\sim 25 \%$ of their cases. Nakamura et al. (2004) found out an appreciable occurrence of the large longitude angles ( $\geq 30$ degree) for the earthward fast flows events. Considerable cross-tail velocity components $\left(\left|V_{y}\right| \sim\left|V_{x}\right|\right)$ were shown in a few case studies (e.g. Sergeev and Lennartsson, 1988; Borodkova et al., 2002). Recently, the average plasma sheet flow pattern in the XY plane was obtained by Hori et al. (2000) and Kaufmann et al. (2001) by averaging the Geotail velocity measurement for a few years. An important feature of this average pattern is the large flow velocity $(100-200 \mathrm{~km} / \mathrm{s})$ in the region $\mathrm{X}<-15 R_{E},-5 R_{E}<Y<10 R_{E}$, where a significant $V_{y}$ component is also observed. Results of Baumjohann et al. (1990) showed a maximal occurrence of the fast flows' in the same region. So, the fast flows contribution to the average pattern, particularly in the cross-tail component, may be significant. To our knowledge, the origin of this transverse flow component in the tail plasma sheet has not been investigated before. 


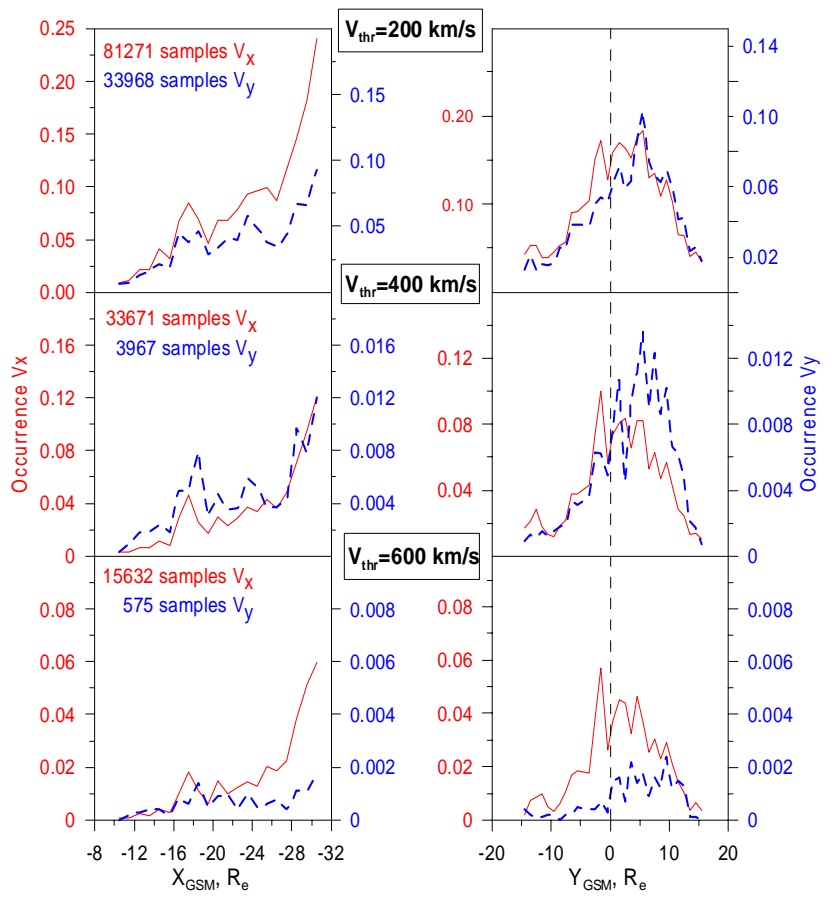

Fig. 1. Occurrence frequency of the $\left|V_{x}\right|>V_{\mathrm{thr}}$ (red) and $\left|V_{y}\right|>V_{\mathrm{thr}}$ (blue) samples, depending on the $\mathrm{X}$ (left column) and $\mathrm{Y}$ (right column) GSM coordinates for three different velocity thresholds (200, 400 and $600 \mathrm{~km} / \mathrm{s}$ ). The number of events for each distribution is indicated.

The aim of the present work is to study the spatial distribution of the large $V_{y}$ ion bulk flows (Sect. 2), the relationship of this transverse component with the BBFs (Sect. 3), and the flow rotation near the BBF front (Sect. 4). The last section provides a summary of results and discussion.

\section{Occurrence of strong $V_{y}$ flows}

In this section each 12-second sample with $\left|V_{y}\right|>V_{\text {thr }}$ (where $V_{\text {thr }}$ is a threshold value) is considered as a separate event. Three threshold values are tried: $V_{\mathrm{thr}}=200,400,600 \mathrm{~km} / \mathrm{s}$. Also all events with $\left|V_{x}\right|>V_{\text {thr }}$ (same threshold) are used to compare the $\left|V_{y}\right|>V_{\text {thr }}$ and $\left|V_{x}\right|>V_{\text {thr }}$ distributions. The occurrence frequency is defined as the ratio of the event number in the certain spatial bin to the number of all 12-second observations in the same area. Our study is based on Geotail observations made between May 1995 and May 1999. Magnetic field components and plasma parameters (12-s averages) are used. Only the measurements in the region $\mathrm{X}<-15 R_{E}$, $-15<\mathrm{Y}<20 R_{E}$ in the inner plasma sheet (plasma parameter $\beta>0.5$ ) are considered.

The occurrence frequency distributions in X and Y GSM coordinates are presented in Fig. 1 for three threshold values. Each point in the right (left) panel shows the $\left|V_{y}\right|>V_{\mathrm{thr}}$ and $\left|V_{x}\right|>V_{\text {thr }}$ occurrence in the $1 R_{E} \mathrm{X}(\mathrm{Y})$ interval for all

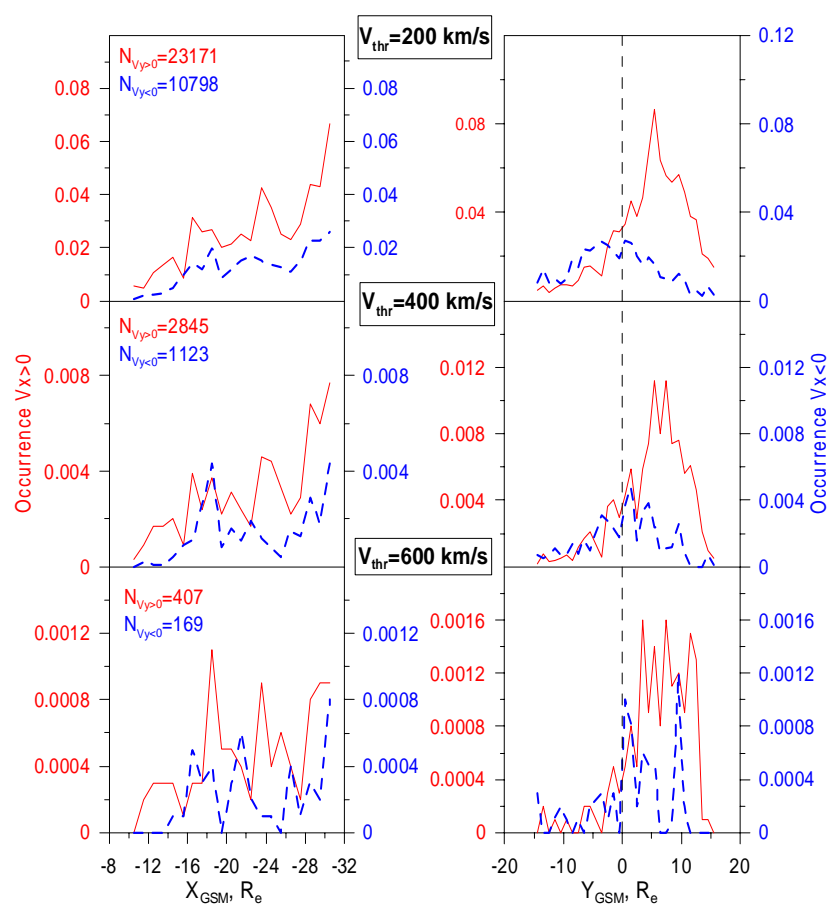

Fig. 2. Occurrence of the $\left|V_{y}\right|>V_{\text {thr }}$ for $V_{y}>0$ (red) and $V_{y}<0$ (blue), depending on the $\mathrm{X}$ (left column) and $\mathrm{Y}$ (right column) GSM coordinates for different velocity thresholds (200, 400 and $600 \mathrm{~km} / \mathrm{s}$ ).

$\mathrm{Y}(\mathrm{X})$ values. Figure 1 shows that $\left|V_{y}\right|$ and $\left|V_{x}\right|$ distributions are similar for each $V_{\text {thr }}$ value, although their probabilities are different: the frequency of large $\left|V_{x}\right|$ values strongly exceeds the frequency of the large $\left|V_{y}\right|$ values (by 2 to 15 times, depending on the threshold value and the observation region). There are some common features of the $\left|V_{y}\right|$ and $\left|V_{x}\right|$ distributions. First, their occurrence increases in the tailward direction, maximizing at the $r \sim 30 R_{E}$ (outer limit of the Geotail orbit used in this study). Second, both have a maximal frequency in the central sector of the tail, the majority of $\left|V_{x}\right|>V_{\text {thr }}$ and $\left|V_{y}\right|>V_{\text {thr }}$ events being observed at $-10 R_{E}<Y<10 R_{E}$. The major difference is the appreciable shift of the $\left|V_{y}\right|$ distribution maximum towards dusk.

To learn more about this dawn-dusk asymmetry we repeated the analysis separately for the $V_{y}<0$ and $V_{y}<0$ subsets. Results are presented in Fig. 2, which shows that the dawn-dusk asymmetry is basically formed due to an excess of $V_{y}>0$ events on the dusk side. The $V_{y}$ histogram in Fig. 3a shows that the $V_{y}>0$ are much more frequent. Such asymmetry may result from a superposition of the symmetric flows with the dawn-to-dusk ion diamagnetic drifts, as it has been previously shown by Hori et al. (2000). Figure $3 b$ illustrates that the distribution can really become symmetric by subtracting $45 \mathrm{~km} / \mathrm{s}$ from all $V_{y}$ values. The exact value slightly depends on the observation point in the tail and on the threshold value. This drift magnitude $\sim 50 \mathrm{~km} / \mathrm{s}$ is similar to the 


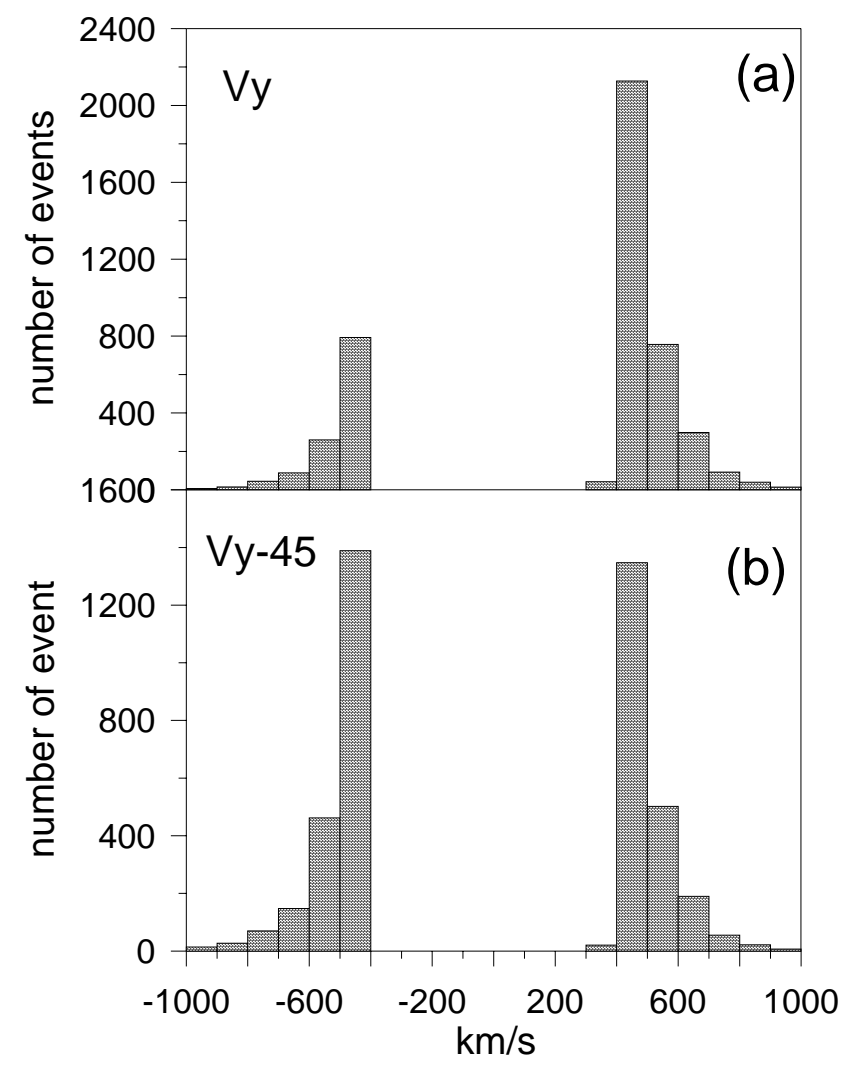

Fig. 3. Histogram of the large cross-tail velocity component occurrence: (a) the original $V y$ distribution (for $|V y|>400 \mathrm{~km} / \mathrm{s}$ ), (b) the drift-shifted distribution of $|V y-45 \mathrm{~km} / \mathrm{s}|$.

$V \sim 40 \mathrm{~km} / \mathrm{s}$ component found by Kaufman et al. (2001) on the duskside, but it is larger than the $V \sim 18 \mathrm{~km} / \mathrm{s}$ obtained by Hori et al. (2000) at $X=-15 R_{E}$ in the midnight meridian.

\section{Relationship of large $V_{y}$ events with the high-speed plasma streams in the tail}

The similarity of large $\left|V_{y}\right|$ and $\left|V_{x}\right|$ flow occurrences suggests a possible relationship of large $\left|V_{y}\right|$ events to the fast $V_{x}$ flows. Our survey of individual events shows that during the isolated $V_{x}$ enhancement above the average level (that is, above several tens $\mathrm{km} / \mathrm{s}$, according to Kaufmann et al., 2001), the $V_{y}$ flows often display a complicated variation at smaller time-scale. In this case the episodes with $V_{y}>V_{\mathrm{thr}}$ are shorter than those with $V_{x}>V_{\text {thr }}$ for the same threshold value. An example is given in Fig. 4, where the interval of $V_{y}>400 \mathrm{~km} / \mathrm{s}$ $(\sim 40 \mathrm{~s})$ is highlighted in green and the $V_{x}>400 \mathrm{~km} / \mathrm{s}$ time interval $(\sim 6 \mathrm{~min})$ is marked by the vertical lines. In these events one typically observes the $\left|V_{x}\right|$ enhancement, the density and pressure reduction and the temperature growth at the beginning of the flow burst, which are the well-known BBF attributes (see Angelopoulos et al., 1994; Ohtani et al., 2004).

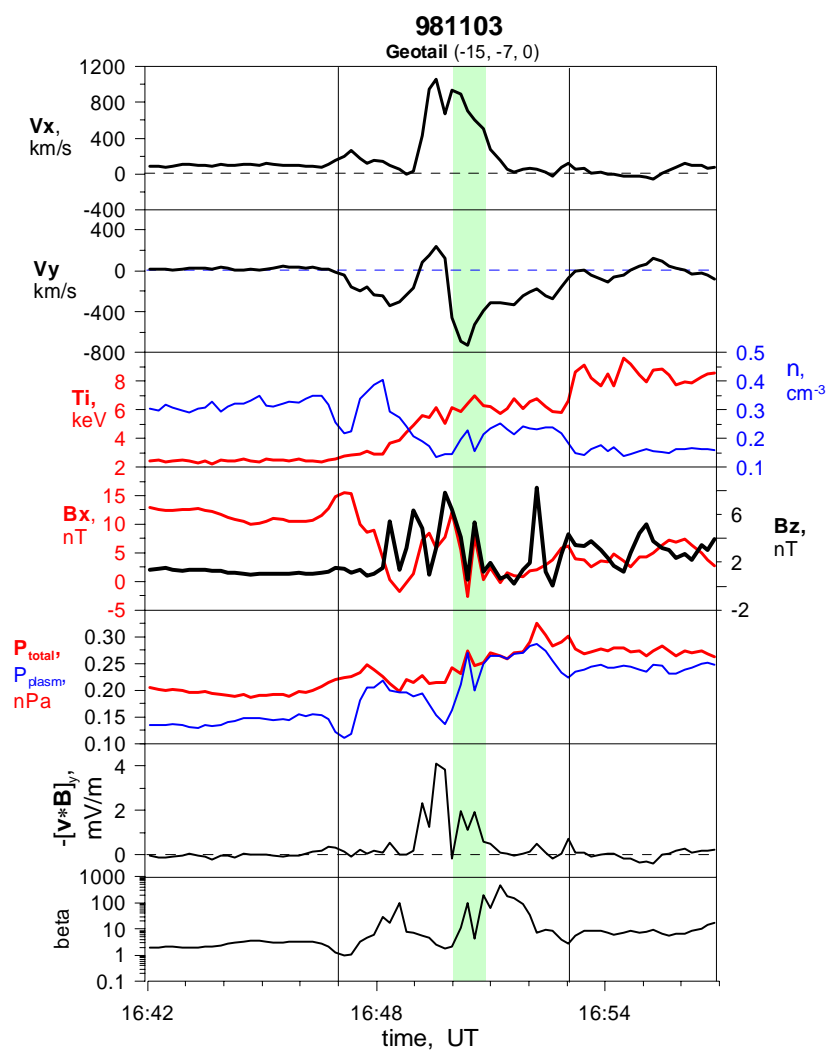

Fig. 4. Variation of the plasma parameters and magnetic field components observed on 4 September 1997. The time interval with $|V y|>400$ is highlighted.

In this section we study the average characteristics of large $\left|V_{y}\right|$ events. The event is here defined as an interval, satisfying the following conditions: (1) $\left|V_{y}\right|_{\max }>400 \mathrm{~km} / \mathrm{s}$., (2) $\beta>0.5$ before the front of the fast stream (that is the BBF is observed in the CPS region). A brief $\beta$ value decrease below 0.5 is allowed inside the event due to strong magnetic field and plasma parameters variations. (3) To obtain a more distinct picture at the front, we consider only those events, which had $\left|V_{y}\right|<200 \mathrm{~km} / \mathrm{s}$ during at least $5 \mathrm{~min}$ before the $\mathrm{BBF}$ onset. The last requirement strongly reduces the number of selected events.

The 98 selected events with $\left|V_{y}\right|>400 \mathrm{~km} / \mathrm{s}$ showed the BBF attributes in $>90 \%$ of the cases. To obtain an average portrait of the large $V_{y}$ events we performed a superposed epoch analysis. A critical point of the superimposed epoch analysis is the choice of the reference time $\left(T_{0}\right)$. As was shown by Ohtani et al. (2004), the results differ depending on the $T_{0}$ choice, whether you use either a crossing of some velocity threshold, or the start of the magnetic field $B_{z^{-}}$ component variation. Below we use the reference time at the start of a sharp $\left|V_{y}\right|$ growth above $200 \mathrm{~km} / \mathrm{s}$. The results are presented in Fig. 5. Note that the average $\left|V_{y}\right|_{\max }$ value does not exceed the $400 \mathrm{~km} / \mathrm{s}$ value. The reason is that different events have different front durations and their $V_{y}$ maxima do 


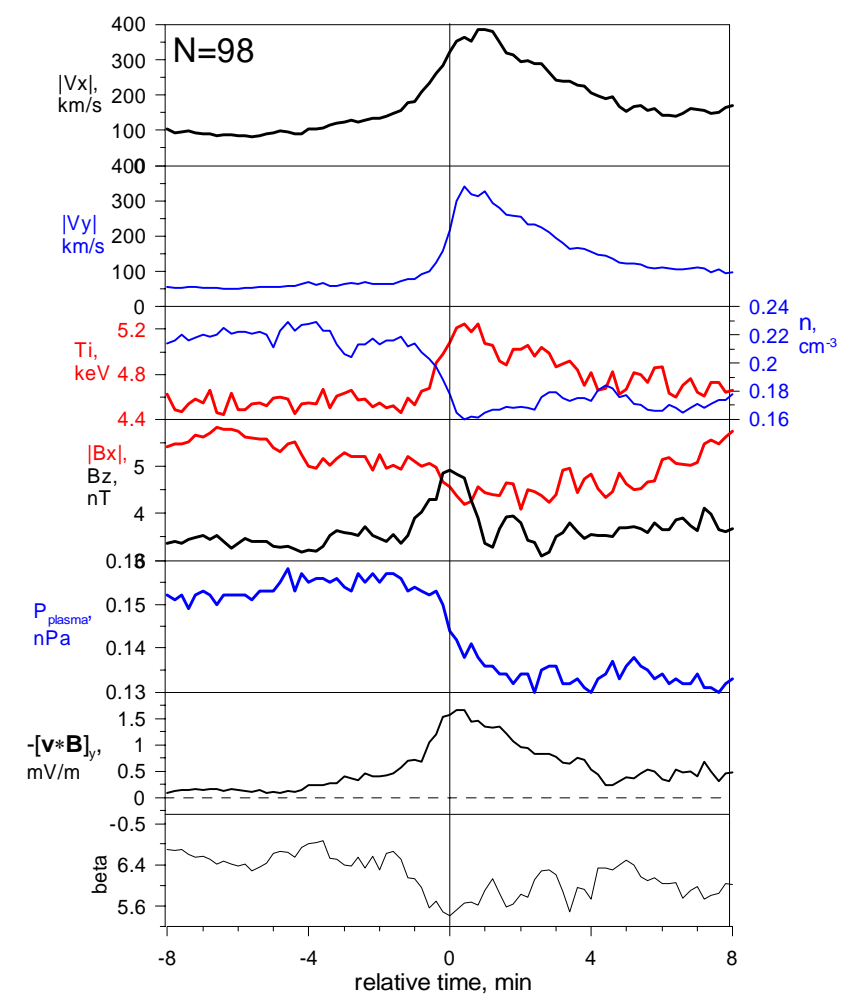

Fig. 5. Average velocity, magnetic field and plasma parameter variations for $\left|V_{y}\right|>400 \mathrm{~km} / \mathrm{s}$ events, resulting from the superposed epoch analysis. Reference time is defined as the start of sharp $\left|V_{y}\right|$ growth above $200 \mathrm{~km} / \mathrm{s}$.

not coincide, so the average picture blurs. Figure 5 confirms the suggestion about a close relationship between the large $V_{y}$ events and the BBFs: the averaged $\left|V_{x}\right|$ peak value exceeds the $\left|V_{y}\right|$ peak value, and the magnetic field and plasma variations are analogous to those obtained in Angelopoulos et al. (1994) and Ohtani et al. (2004) for the BBFs.

The analysis of the large $V_{y}$ and $V_{x}$ spatial distributions in the previous section showed that for the fixed $V_{\text {thr }}$ the probability of large $\left|V_{y}\right|$ values is much smaller than that of large $\left|V_{x}\right|\left(\sim 10\right.$-fold for $\left.V_{\mathrm{thr}}=400\right)$. Does it mean that only a small part of the BBFs has a large $V_{y}$-component, or is this because the $\left|V_{y}\right|>V_{\text {thr }}$ events have a smaller space-time scale as compared to the BBFs? What is the ratio of the maximum $\left|V_{x}\right|$ and $\left|V_{y}\right|$ values in the fast flows in the plasma sheet?

To obtain the answers we studied the BBFs (all intervals with $\left|V_{x}\right|>400 \mathrm{~km} / \mathrm{s}$, in total -1022 isolated events), using the same criteria as applied above for the large $\left|V_{y}\right|$ events. After many attempts, we present here (Fig. 6) the average characteristics obtained by the superposed epoch analysis with a reference time, corresponding to the plasma pressure maximum during a 1-min interval just before the sharp $\left|V_{x}\right|$ growth. In our opinion this epoch time may correspond to the boundary between the background plasma, grabbed at the BBF front, and the depleted plasma flux

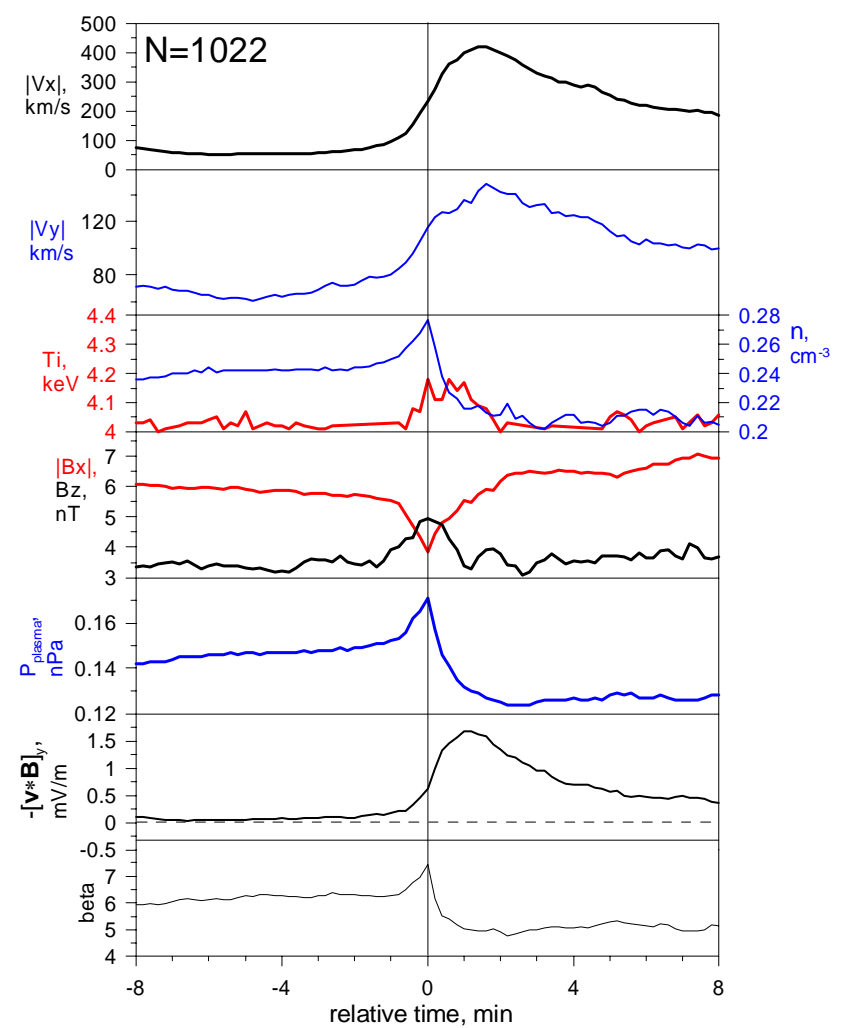

Fig. 6. The same as in Fig. 5 for events with $\left|V_{x}\right|>400 \mathrm{~km} / \mathrm{s}$.

tube (the BBF proper). The average picture of the BBFassociated plasma parameters and magnetic field variations is similar to that obtained by Ohtani et al. (2004) in all aspects, except the $V_{y}$ component magnitude. According to our data $\left|V_{y}\right|>400 \mathrm{~km} / \mathrm{s}(300 \mathrm{~km} / \mathrm{s})$ is observed for, respectively, $21 \%$ (43\%) of all events when $\left|V_{x}\right|>400 \mathrm{~km} / \mathrm{s}$. Moreover, the events for which $\left|V_{y}\right|$ is small $(<100 \mathrm{~km} / \mathrm{s})$ are rare $(\sim 3 \%)$. Average duration of the time interval when $\left|V_{y}\right|$ exceeds $200 \mathrm{~km} / \mathrm{s}$ is $\sim 3 \mathrm{~min}$ (Fig. 5). The corresponding time interval for $\left|V_{x}\right|$ is $\sim 6$ min (Fig. 6). The average ratio $\left(R=\left|V_{y}\right|_{\max } /\left|V_{x}\right|_{\max }\right)$ between $\left|V_{x}\right|$ and $\left|V_{y}\right|$ peak values for all events with $\left|V_{x}\right|>400 \mathrm{~km} / \mathrm{s}$ was found to be $R=0.45$.

\section{Plasma flow structure near the BBFs' front}

As follows from the theoretical considerations, the bubble polarization corresponds to a two-vortex plasma motion in the bubble and surrounding plasma. Near the bubble leading front it has a plasma velocity component transverse to the tail axis, whose value is expected to be maximal at the leading flux edge (in simple models), and whose sign coincides with the sign of the y-component of the outer normal to the bubble front. The streamlines are similar to those in the flow around the obstacle. 


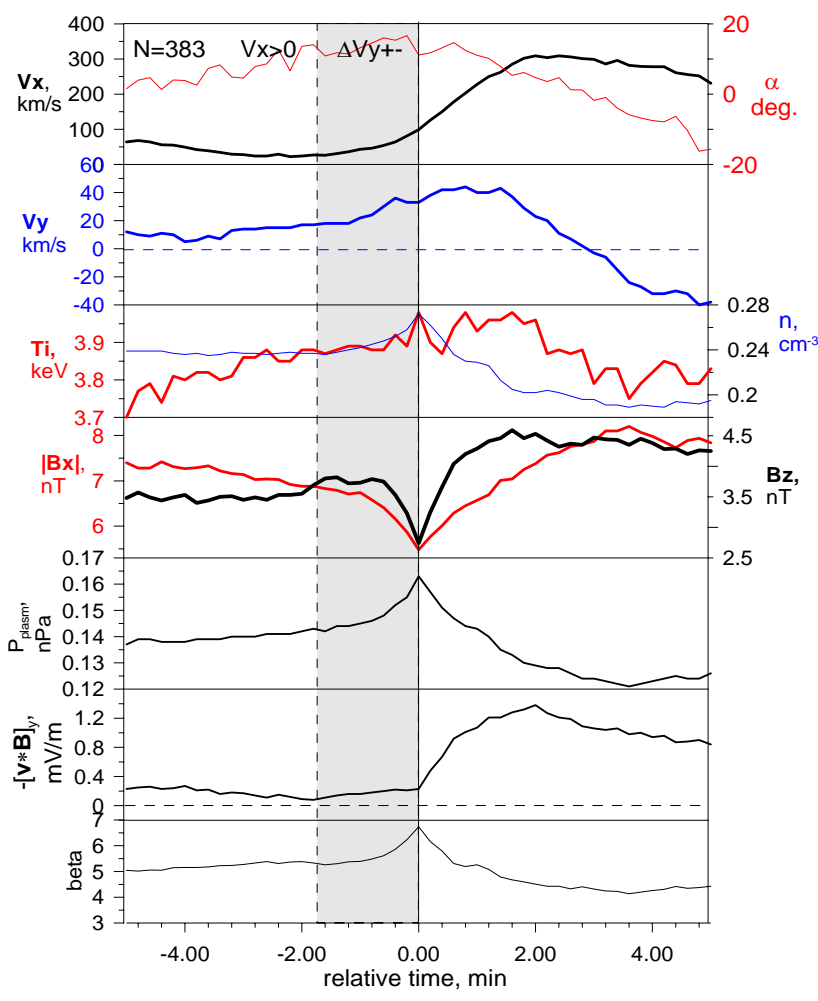

Fig. 7. Average velocity, magnetic field and plasma parameter variations for one group of the earthward moving fast flows. Reference time is defined as the time of the plasma pressure maximum.

To study the flow morphology near the BBF front, all 1022 events found in the previous section were divided into 4 groups. First of all, they were separated by the $V_{x}$ flow direction (818 earthward flows and 204 tailward flows). Besides, most of the events were found to display the change in the $V_{y}$ sign; see e.g. Fig. 4. Therefore, each group was split into two groups, depending on the $V_{y}$ sign change: from positive to negative or vice versa. Afterwards, the average portrait of each group was obtained, using a superimposed epoch analysis, and the relationship between the times of $V_{y}$ maxima and minima was also studied.

We are reminded that the $T_{0}$ value in the superimposed epoch analysis was defined at the plasma pressure maximum. The result is illustrated in Fig. 7 for one group of events (with $V_{x}>0$ and $V_{y}$ changing sign from positive to negative). Results for the other groups of events are similar in main features. Besides the typical BBF characteristics, two interesting features should be noted. First, one can see in Fig. 7 the region of BBF interaction with the surrounding plasma (highlighted), which is characterized by a smooth increase in velocity, density, plasma pressure and electric field before the crossing of a sharp BBF leading front (at $T_{0}$ ). The average time scale is $1-2 \mathrm{~min}$, for the average plasma velocity $\sim 70-150 \mathrm{~km} / \mathrm{s}$ it gives the size of this interaction region $\sim 1-$ $3 R_{E}$. The second feature is the $V_{y}$ variation. The absolute
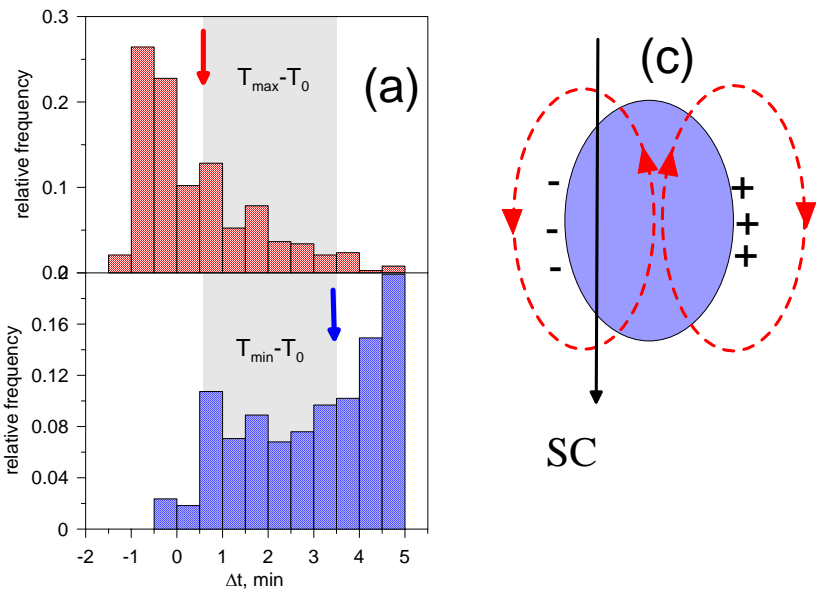

SC

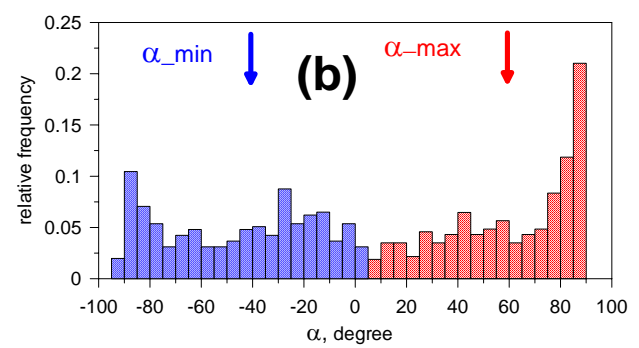

Fig. 8. (a) Histograms of the time delay of the maximal velocity deviation to dusk (top) and to dawn (bottom) relative to the time of BBFs' front pass; (b) histograms of the maximal velocity deviation values: to dawn (left) and to dusk (right); (c) the scheme of the plasma motion (red vortexes) around BBF (blue oval) in the XY plane.

$V_{y}$ value increases in the interaction region, $V_{y}$ reaches its first extreme near the front, then decreases, changes its sign and reaches the second extreme somewhere in the BBF trailing part. The trailing part may be roughly determined based on the average BBF duration of about ten minutes. Note that a similar $V_{y}$ variation would be observed when crossing the vortex. The vortex-like variation is more clear when considering the angle of the $\mathrm{V}$ vector deviation $(\alpha)$ from the $\mathrm{X}$ axis (the red line in the upper panel of Fig. 7, corresponding to the right vertical axis).

In Fig. 7 the $\alpha$ value is underestimated due to the large scatter in the relative observation time and duration of the angle variations. To find the real $\alpha$ values and the location of its extremes relative to the BBF front, for each event we determined the maximum and minimum $\alpha$ values and corresponding times in the time range $\left(T_{0}-1 \mathrm{~min}, T_{0}+5 \mathrm{~min}\right)$. This statistics is presented in Fig. 8 for one group of earthward BBF events. Similar results were found for other groups of events. The upper (center) panel presents the distribution of $\alpha$ maxima (minima) times, and the median values are shown by the arrows. The bottom panel demonstrates the distributions of $\alpha_{\max }$ and $\alpha_{\min }$ values. According to these data the first $\alpha$ extreme is observed near the BBF front, being 


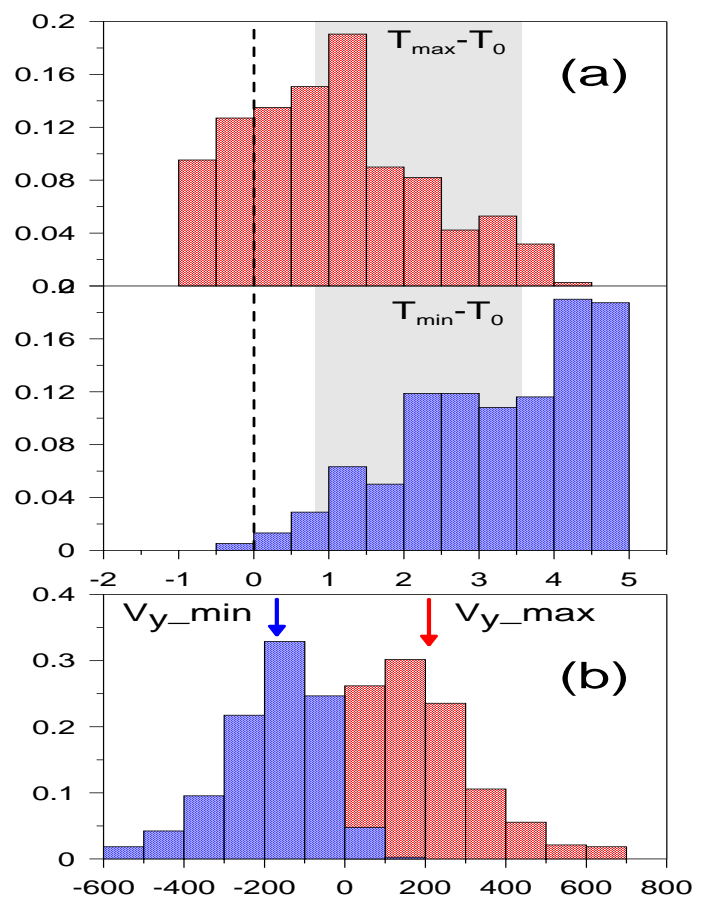

Fig. 9. (a) Histograms of the time delay of the maximal (top) and minimal (bottom) $V_{y}$ velocity value relative to the time of the BBFs' front pass (dashed line); (b) histograms of the minimal (left) and maximal (right) $V_{y}$ value.

somewhat shifted inside the flows on average. The time delay between the first and second extremes is about $3 \mathrm{~min}$ (highlighted by the shaded band), giving a distance estimate $\sim 7 R_{E}$, comparable with the BBF scale in the $\mathrm{X}$ direction for the average plasma speed inside the BBF $\sim 250 \mathrm{~km} / \mathrm{s}$. The extreme $\alpha$ values vary in a broad range without clear peak. This can be due to different spacecraft locations relative to the BBFs central line (see the scheme in Fig. 8). Though $\alpha$ extremes, on average, are observed near the leading and back BBF fronts, they are frequently reached inside the BBF. Particularly, about half of the events are in the time range between the first and second extremes, inside the color band in Fig. 8.

Note that the large $\alpha$ value may result from a small $V_{x}$ outside the fast flow rather than from a large $V_{y}$ value. To test the relationship between extremes $\alpha$ and $V_{y}$ we analyzed $V_{y \max }$ and $V_{y \text { min }}$ values and their registration times in the same way as it was done above for the $\alpha_{\max }$ and $\alpha_{\min }$ values. The result of this analysis (Fig. 9) looks very similar to the previous result for $\alpha$ : the first $V_{y}$ extreme is observed near the BBF front, the average time delay between the first and second extremes is about $3 \mathrm{~min}$. The median value $V_{y \max }$ $\left(V_{y \min }\right)$ is $202 \mathrm{~km} / \mathrm{s}(-180 \mathrm{~km} / \mathrm{s})$.

The study of tailward BBFs $\left(V_{x}<0\right)$ showed similar velocity components variations - Fig. 10. However, there are some differences. In particular, the plasma density grows

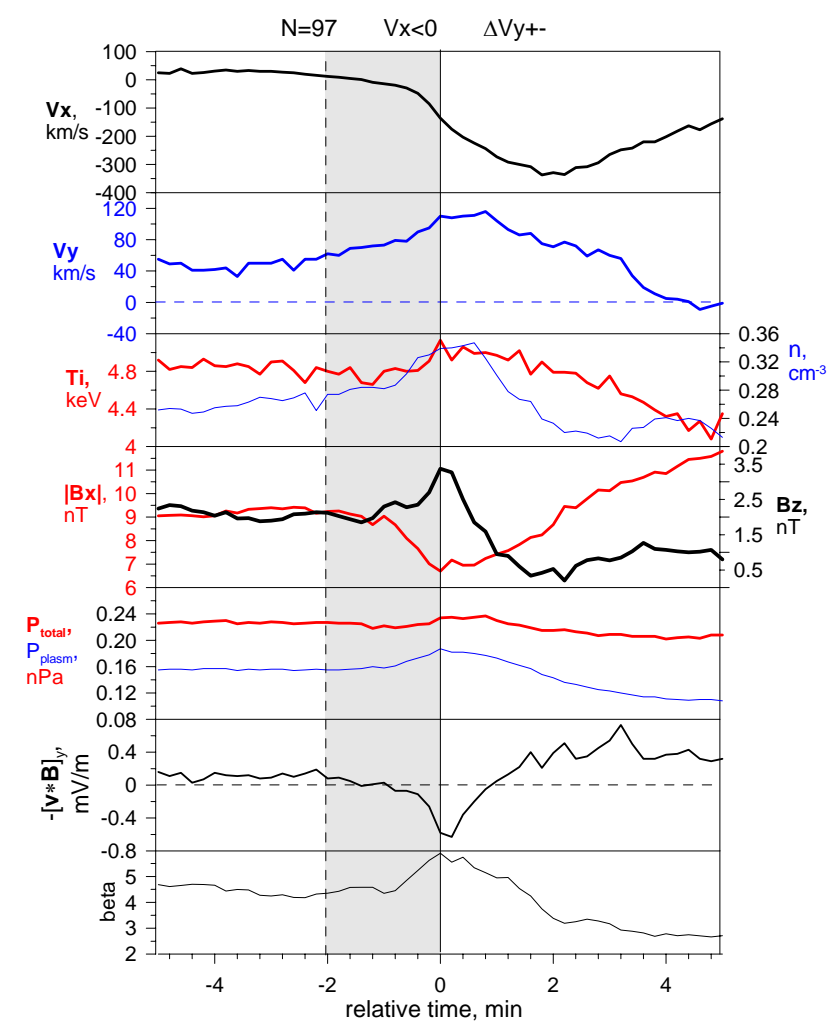

Fig. 10. The same as in Fig. 7 for one group of the tailward moving fast flows.

not only before the leading BBF front, but also during some time after its crossing, different from the earthward flows. The density change in both cases is about $20 \%$ of the background value. Note that according to the Pontius and Wolf (1990) bubble scenario, the tailward flows should be denser than the surrounding plasma, and their electric field should be negative. Our results show that near the front of a tailward flux a bipolar $E_{y}=-[\boldsymbol{V} \times \boldsymbol{B}]_{y}$ variation is observed: a negative pulse lasting about 2 min with a minimum near the BBF front followed by the $E_{y}$ reversal. The amplitudes of the positive and negative pulses are approximately the same $( \pm 0.5 \mathrm{mV} / \mathrm{m})$, being about one-third of their peak value in the earthward BBFs.

The $V_{y}$ extreme values and their observation time relative to the BBF front are basically the same in the tailward and earthward flows, excluding the value of the ratio $\left|V_{y}\right|_{\max } /\left|V_{y}\right|_{\min }$. For tailward flows this ratio is about 2.8, as compared to $\sim 1.3$ for the earthward ones.

\section{Discussion}

Results of our statistical analysis give quite convincing evidence that the majority of the magnetospheric plasma sheet fast flows have the $V_{y}$-component that is, on average, one half as large as the main $V_{x}$ component. The median 
$\left|V_{y}\right|_{\max } /\left|V_{x}\right|_{\max }=0.47$ ratio obtained in the present study gives the average angle of velocity vector deviation from the tail axis equal to 25 degrees. This value agrees with that obtained by Baumjohann et al. (1990) for distances less than $20 R_{E}$. As follows from our results (Figs. 5 and 6), this angle is not constant during the BBF passage. The variation of the velocity angle resembles the variation expected during the passage of a plasma vortex, which is predicted by the bubble model of fast flows (Pontius and Wolf, 1990; Birn et al., 2004). According to it, we should observe a clockwise velocity vector rotation on the dawn side of the BBF, and an anticlockwise rotation on its dusk side. So the sign of the $Y$ component of the plasma velocity near the leading edge of the BBF should coincide with the sign of the Y component of the outward normal, being negative at the BBF dawn side and positive at its dusk side. Such a correspondence was found in several cases by Sergeev et al. (1996). In fact, the flow structure near the BBF front is usually more complicated than in the idealized model considered by Pontius and Wolf (1990).

We have only one observation point, so we do not know whether we are at the dawn or dusk side of the fast flow in each event. However, when analyzing such a large number of events (1022 events in our analyses) we believe that the spacecraft position at the dawn or dusk sides of the BBF are equiprobable. If our interpretation of the $V_{y}$ variation as the feature of the passing plasma vortex is correct, we would observe the clockwise variation for a dawnward spacecraft position (like in 436 events in the earthward flows.) and anticlockwise for the duskward position (as in 382 events) The corresponding numbers for tailward flows are 97 and 107. So the probability to observe both kinds of rotation is nearly the same, corresponding to the equal probability to pass through the fast flows at the dawn or dusk side.

As an additional test we used the results of Nakamura et al. (2001), where the normal to the BBF frontal boundary was determined for 14 events using the Geotail data. For all events from their list we considered the sign of the cross-tail $V_{y}$-component near the BBFs' front, using the plasma pressure peak as an indication of the BBF front crossing. In this comparison for 13 events the signs of the $V_{y}$ and $n_{y}$ components agree with each other. In the single (opposite sign) event the $n_{y} / n_{x}$ ratio was $\sim 0.2$ (suggesting the measurements made near BBF center) as compared to its value between 0.5 and 1.5 for 13 remaining events.

According to our results, the spatial size of the considered typical $V_{y}$ velocity disturbance along the $\mathrm{X}$-axis is $\sim 7-$ $10 R_{E}$. That's why the BBF-associated $V_{y}$-component discussed here cannot be a manifestation of the local, smallscale plasma sheet irregularities.

Based on the obtained results we suppose that a considerable part of observed large $V_{y}$ plasma velocity component is connected with the passage of a polarized flux tube (BBF), causing the vortex plasma motion.

The pattern of plasma motion, obtained here, differs from the theoretical model in one important point. According to our data, the plasma behind the BBF front is also involved in the vortex motion, together with the background plasma. Indeed, the extreme $\alpha$ values are often observed inside the BBF but not near its front. Partly this may be due to the complex BBFs structure, since usually we do not observe isolated high-velocity pulses, but a superposition of interacting flows. So the former BBF is the background for the next one, in that case the vortex motion may arise from (and be modified by) their collision.

Similar average magnetic field and plasma variations are observed for all velocity thresholds $(200,400$ and $600 \mathrm{~km} / \mathrm{s})$, with the occurrence frequency of fast flow observation quickly growing with a decrease in the threshold value. The velocity variation at the $\mathrm{BBF}$ front is similar to the obstacle streamline. Note that in the majority of the events we can observe the plasma vortex clearly only near the leading BBFs' front. The whole vortex can be seen unperturbed only during the passage of very rare short isolated flows. In reality, we often observe a long complicated variation resulting from the interaction of different individual fast flows passing at different trajectories and having different $V_{x}$ peak velocities. Note that in the majority of events we can observe the plasma vortex clearly only near the leading BBFs' front, where the vortex-associated flow tends to be directed toward the flanks. Besides, the main convection component in the middle tail during the quiet periods (without BBFs) is the dawn-dusk drift velocity. Taking into account the substantial contribution of the fast flows to the convection, we can expect that the vortex plasma motion, accompanying the BBFs, plays a significant role in the formation of a cross-tail component of the average convection pattern (Hori et al., 2000; Kaufmann et al., 2001).

\section{Conclusions}

A significant part of the observed cross-tail plasma velocity component can be understood as a result of the fast flows' interaction with the background plasma and with each other. The scale of the interaction region at the leading front is about 1-3 $R_{E}$. Large $V_{y}$ velocity component probably arises from vortex plasma motion in the vicinity of the BBF front, which may be explained based on the model of the polarized plasma bubbles. The average vortex size in the direction of propagation is about $10 R_{E}$.

Acknowledgements. We thank V. Sergeev, M. Shukhtina and E. Timofeev for helpful comments. The work was partially supported by INTAS grant No 03-51-3738 and RF Ministry of Education grant "Intergeophysics" No 2.2.2.2.2190. RFBR grant 07-0291703.

Topical Editor I. A. Daglis thanks K. Maezawa and another anonymous referee for their help in evaluating this paper. 


\section{References}

Angelopoulos, V., Baumjohann, W., Kennel, C. F., et al.: Bursty bulk floes in the inner central plasma sheet, J. Geophys. Res., 97, 4027-4039, 1992.

Angelopoulos, V., Kennel, C. F., Coroniti, F. V., Pellat, R., Spence, H. E., Kivelson, M. G., Walker, R. J., Baumjohann, W., Feldman, W. C., Gosling, J. T., and Russell, C. T.: Characteristics of ion flow in the quiet state of the inner plasma sheet, Geophys. Res. Lett., 20, 1711-1714, 1993.

Angelopoulos, V., Kennel, C. F., Coroniti, F. V., et al.: Statistical characteristics of bursty bulk flow events, J. Geophys. Res., 99, 21, 257-21, 280, 1994.

Angelopoulos, V., Coroniti, F. V., Kennel, C. F., et al.: Multipoint analysis of a bursty bulk flow event on 11 April 1985, J. Geophys. Res., 101, 4667-4689, 1996.

Baumjohann, W. and Paschmann, G.: Characteristics of high-speed ion flows in the plasma sheet, J. Geophys. Res., 95, 3801-3809, 1990.

Baumjohann, W.: Modes of convection in the magnetotail, Phys. Plasmas, 9, 3665-3667, 2002.

Birn, J., Raeder, J., Wang, Y. L., et al.: On the propagation of bubbles in the geomagnetic tail, Ann. Geophys., 22, 1773-1786, 2004,

http://www.ann-geophys.net/22/1773/2004/.

Borodkova, N. L., Yahnin, A. G., Lion, K., et al.: Plasma sheet fast flows and auroral dynamics during substorm: a case study, Ann. Geophys., 20, 341-347, 2002,

http://www.ann-geophys.net/20/341/2002/.

Chen,C. X. and Wolf, R. A.: Interpretation of high-speed flows in the plasma sheet, J. Geophys. Res., 21, 409-418, 1993.
Hori, T., Maezawa, K., Saito, Y., et al.: Average profile of ion flow and convection electric field in the near-Earth plasma sheet, Geophys. Res. Lett., 27, 1623-1626, 2000.

Kaufmann, R. L., Bryan, M. B., Paterson, W. R., et al.: Plasma sheet thickness and electric current, J. Geophys. Res., 106, 6179-6193, 2001.

Nakamura, R., Baumjohann, W., Schödel, R., et al.: Earthward flow bursts, auroral streamers, and small expansions. J. Geophys. Res., 106, 10791-10 802, 2001.

Nakamura, R., Baumjohann, W., Moukis, C., Kistler, L. M., Runov, A., Volwerk, M., Asano, Y., Vörös, Z., Zhang, T. L., Klecker, B., Rème, H., and Balogh, A.: Spatial scale of high-speed flows in the plasma sheet observed by Cluster, Geophys. Res. Lett., 31, L09804, doi:10.1029/2003GL019558, 2004.

Ohtani, S., Shay, M. A., and Mukai, T.: Temporal structure of the fast convective flow in the plasma sheet: comparison between observations and two-fluid simulations, J. Geophys. Res., 109, A03210, doi:10.1029/2003JA010002, 2004.

Pontius Jr., D. H. and Wolf, R.A.: Transient flux tubes in the terrestrial magnetosphere, Geophys. Res. Lett., 17, 49-52, 1990.

Schödel, R., Baumjohann, W., Nakamura, R., et al: Rapid flux transport in the central plasma sheet, J. Geophys. Res., 106, 301313, 2001.

Sergeev, V. A. and Lennartsson, W.: Plasma sheet at $\approx 20$ Re during steady magnetospheric convection, Planet. Space Sci., 36, 353370, 1988.

Sergeev, V. A., Angelopoulos, V., Gsling, J. T., et al.: Detection of localized, plasma-depleted flux tubes or bubbles in the midtail plasma sheet, J. Geophys. Res., 101, 10 817-10 826, 1996. 